\title{
Vibrational contributions to phase stability in the Mo-Ru system
}

\author{
Sean H. Kessler, David G. Abrecht, Richard A. Clark, Jon M. Schwantes
}

National Security Directorate, Pacific Northwest National Laboratory, 902 Battelle Boulevard, Richland, WA 99352

Density functional theory using the Perdew-Burke-Ernzerhof functional and the small displacement method was used to calculate the quasiharmonic phonon density of states (DOS) for 69 ordered structures in the Mo-Ru substitutional alloy system to estimate the effect of vibrational contributions to the free energy of formation. These structures were used to determine interaction parameters in the cluster expansion approximation up to triplet sets to incorporate the vibrational contribution into the configurational energy of formation for the randomly mixed system. The free energies calculated using this approximation are shown to resolve lingering disagreement between reported theoretical and experimental results, and the stabilizing effect of vibrational entropy is observed to improve theoretical predictions of the Mo-Ru phase diagram.

\section{Introduction}

Noble metal inclusions in nuclear fuel have traditionally been difficult to characterize because of the small quantities of species involved, the radioactive environment, and the unresolved question of the mechanism by which the particles are formed [1-3]. Because of the difficulties involved in performing measurements in highly radioactive systems, theoretical examinations using $a b$ initio simulations of the metallic phases in question can provide a great deal of insight into the thermodynamics guiding particle precipitation. A detailed understanding of the Mo-Ru substitutional alloy system is important for an evaluation of the behavior of metal inclusions in irradiated nuclear fuels, which typically contain molybdenum and ruthenium in the highest quantities (with other significant elements being technetium, palladium, and rhodium) [3-6]. In this work, we examine an approach to density functional theory descriptions of the Mo-Ru substitutional alloy system that retains the disordered nature of the modeled solid solution while incorporating vibrational effects in calculating the free energy of formation.

Simulations based on density functional theory (DFT) techniques have successfully reproduced phase diagrams for solid solutions of several transition metals and have been used in the past to attempt to describe the Mo-Ru system [7-11]. Recent work reported by Middleburgh et al. has even had success probing the energetic behavior of random configurations of the five-metal alloy [12]. Such studies, however, have been limited by the need to reconcile predicted thermodynamic properties with those obtained through direct experiment in order to validate the results of electronic structure calculations. For the hexagonal close-packed (hcp, commonly referred to as the $\varepsilon$ phase) Mo-Ru system, significant discrepancies remain between experimental and theoretical results, particularly in the calculation of formation enthalpies [13]. Previous work has suggested that this discrepancy may be attributed to differences in the hcp-bcc lattice stability of Mo (represented by the change in enthalpies between the two configurations, $H_{\mathrm{Mo}}^{h c p}-H_{\mathrm{Mo}}^{b c c}$ ) as calculated through ab initio methods versus the value recommended by CALPHAD models $[10,14]$. The possibility of increased ordering has also been investigated through means of a cluster expansion and ground-state search, although the gap between experimental and theoretical enthalpy of formation is still not fully resolved in that case [9].

In our work described below, the cluster expansion method, which is well-established for determining the configurational energy of binary alloys [15-18], is extended through the coarse-graining framework to include both configurational and vibrational contributions to the free energy [19-23]. We 
demonstrate that this additional vibrational contribution has a significant impact in driving the phase stability of the Mo-Ru system.

\section{Computational Method}

The cluster expansion approximation is a technique for evaluating a thermodynamic property of a random alloy material by describing the random configuration as a series of ordered cluster sets, based on the generalized Ising model $[15,20,21]$. The usual framework of the expansion involves the expression of the property of interest (here, the free energy) for a given configuration $\boldsymbol{\sigma}$ at a given temperature $T$ :

$$
F(\sigma, T)=\sum_{\alpha} \Omega_{\alpha} \xi_{\alpha}(\sigma) J_{\alpha}(T)
$$

where for each cluster $\alpha, \Omega_{\alpha}$ is the multiplicity (or degeneracy) of the cluster, the correlation function $\xi_{\alpha}=\left\langle\prod_{i \in \alpha} \sigma_{i}\right\rangle$ is the average over all symmetrically equivalent clusters of the product of Ising spin values, and the temperature-dependent coefficient $J_{\alpha}(T)$ is called the effective cluster interaction (ECI) [21]. In our model of the Mo-Ru system, the spin values are assigned as $\sigma_{\mathrm{Ru}}=1$ for each $\mathrm{Ru}$ atom and $\sigma_{\mathrm{Mo}}=-1$ for each Mo atom.

In principle, an exact description of the energy of a system is possible for any stable configuration if a full set of ECI's is provided for every cluster in the lattice $[16,17]$. In practice, sufficiently accurate results can be achieved through truncation of the expansion to lower-order clusters whose longest interatomic distance are within a preselected cutoff value. A training set of structures is chosen to span the concentration range of interest for several ordered configurations, and the energies of this set are determined through electronic structure calculations. ECI values can then be obtained through the structure inversion method, which entails a least-squares fit to Eq. (1) at each chosen temperature. With the values of $J_{\alpha}$ derived in this manner, an energy of formation can be computed quickly for any chosen configuration.

The cluster expansion approach has the advantage over both the coherent potential approximation (CPA, used to determine the enthalpy of formation) and the virtual crystal approximation (VCA, used to determine vibrational free energy) that it allows for local relaxation of the system in the process of determining $F(\sigma, T)$ [21]. The effect of local relaxations on energy calculations may be significant in systems for which dynamic instability and atomic size mismatch prevent the description of the atomic behavior in terms of a single fixed lattice [14, 19]. By excluding those structures observed in the process of ionic relaxation to exhibit unstable modes - through either failure to converge, convergence to a different type of lattice from the starting lattice, or calculation of imaginary phonon frequencies in the relaxed structure - we are able to restrict the analysis to physically realizable structures and bypass a description of an unphysical lattice stability $[10,14]$. Results are compared with existing theoretical and experimental thermodynamic values, where available, in order to validate the selected approach.

The structures used in the training set were generated using the Alloy Theoretic Automated Toolkit (ATAT) [24-26]. The bcc $\beta$ phase consisted of compositions in the range $x_{\mathrm{Ru}}=0$ to 0.375 , above which structures were observed to become predominantly unstable. Likewise, the hcp $\varepsilon$ phase spanned compositions from $x_{\mathrm{Ru}}=0.375$ to 1 . Values of $\xi_{\alpha}$ were determined through ATAT based on the ideal lattice for all pair clusters within a cutoff distance of $6 \AA$ in the hcp case and $7.7 \AA$ in the bcc case. Likewise, 
triplet clusters were determined within a cutoff of $4.5 \AA$ in both cases. These selected cutoff values provided 10 symmetrically inequivalent clusters on the bcc lattice and 19 clusters on the hcp lattice. Quaternary clusters, as well as binary and ternary clusters beyond the cutoffs described above, were evaluated to determine their significance on the fit to Eq. (1), but these did not appear to improve the fit visibly and in some cases led to scaling issues in the linear least-squares solution. In total, 20 bcc structures (1-12 atoms in each structure) and 49 hcp structures (2-10 atoms each) were used in the final fit. Monte Carlo simulations were also carried out on supercells with an enclosed radius of $20 \AA$, using the ATAT package, to ensure that the system achieves a disordered state for temperatures above $1000 \mathrm{~K}$ [27].

DFT calculations were carried out within the Vienna Ab-initio Simulation Package (VASP) [28, 29], using the projector augmented-wave (PAW) method and the exchange-correlation energy approximation of Perdew, Burke, and Ernzerhof (PBE) [30, 31]. The plane-wave cutoff energy was set to $350 \mathrm{eV}$ for all structures, and a $13 \times 13 \times 13$ Monkhorst-Pack k-mesh, centered at the $\Gamma$ point, was likewise used across the board [32]. This mesh size was chosen following an investigation of pure Mo (with a one-atom unit cell), which showed that changing the mesh from $7 \times 7 \times 7$ to $13 \times 13 \times 13$ led to a $30 \mathrm{meV}$ difference in reported energy, while increasing the mesh further to $17 \times 17 \times 17$ changed the final energy value by $<1$ $\mathrm{meV}$. Electronic structure calculations in the ionic relaxation steps used a convergence criterion of $10^{-11}$ $\mathrm{eV}$, and ionic relaxations themselves were performed until all Hellmann-Feynman forces on the atoms had magnitudes less than $5 \times 10^{-7} \mathrm{eV} \AA^{-1}$. These criteria were chosen to be suitably strict so as to enable very accurate force-matrix calculations in the determination of the phonon density of states (DOS).

Fully-relaxed structures were converted to supercells, which typically comprised 4 unit cells for a total of 12 to 20 atoms each. Differences in supercell size within this range did not appear to have a significant effect on calculated force constants. Additionally, a $2 \times 2 \times 2$ supercell of pure Mo (8 atoms) was compared with a $3 \times 3 \times 3$ supercell ( 27 atoms), with no significant difference in the final computed entropy. These supercell sizes therefore provide a reasonable means for estimating the vibrational thermodynamic values. Forces on all atoms in each structure were calculated for displacements of $0.04 \AA$ from the relaxed positions, this time at the less stringent convergence criterion of $5 \times 10^{-9} \mathrm{eV}$ for the purpose of conserving computing time. Forces calculated in this manner were nearly identical (with deviations $<0.1 \%$ ) to those calculated at stricter tolerance values in several test cases. Phonon density of states, as well as vibrational entropy and free energy, were calculated using the PHON software [33]. Finally, the lattice vectors of each relaxed structure were expanded isotropically in increments of $0.5 \%$ up to $2 \%$ expansion in order to estimate changes to the configurational and vibrational energies in the quasiharmonic approximation.

\section{Results and Discussion}

\subsection{Ionic Relaxations and Energy of Formation}

The total free energy of formation of a disordered solid solution has three main contributions: configurational, vibrational, and electronic. Assuming that excited electronic states are negligible in this instance leads, for a given temperature $T$, to the expression

$$
\Delta^{f} F=\Delta^{f} E_{c f g}+\Delta^{f} E_{v i b}-T\left(\Delta^{f} S_{c f g}+\Delta^{f} S_{v i b}\right)
$$

By convention, the configurational term $\Delta^{f} E_{c f g}$ is calculated from the difference in ground-state electronic energy between a given structure and the respective pure phases (i.e. hcp $\mathrm{Ru}$ and bcc Mo). The configurational entropy of a perfectly random disordered phase is 


$$
\Delta^{f} S_{c f g}=-k_{B}[x \ln x+(1-x) \ln (1-x)]
$$

for a given fraction $x$ of Ru atoms. The two vibrational terms are described in more detail in the next section.

The configurational energies of formation for each of the $20 \mathrm{bcc}$ structures and 49 hcp structures are shown in Fig. 1. These energies are fitted to a cluster expansion, which is identical to Eq. (1) for the special case in which vibrational terms are neglected and the temperature is set to $0 \mathrm{~K}$. To model the disordered state, we use the Bragg-Williams approximation, such that for a completely random configuration, the $n^{\text {th }}$-order correlation function for any cluster, irrespective of the choice of constituent lattice points, is given by

$$
\xi_{\alpha}^{[n]}=\left[x \sigma_{\mathrm{Ru}}+(1-x) \sigma_{\mathrm{Mo}}\right]^{n}=(2 x-1)^{n},
$$

using the assignment of spin variables mentioned above. Because the largest clusters evaluated here are triplets, the energy is subsequently expressed as a third-order polynomial:

$$
E=\sum_{n=0}^{3} J^{[n]}(2 x-1)^{n},
$$

where $J^{[n]}=\sum_{\alpha} \Omega_{\alpha} J_{\alpha}$ is the sum over all $n^{\text {th }}$-order clusters of the degeneracy-weighted ECI values. The estimated formation energy of the disordered phase is plotted in Fig. 1 as solid lines for both the bcc and hcp lattices, and the corresponding values of $J^{[n]}$ are listed in Table I. Monte Carlo simulations performed at $1000 \mathrm{~K}$ for both phases at all concentrations yielded correlation functions within $5 \%$ of the disordered values predicted by Eq. (4) and energy values within $<1 \%$ of those predicted by Eq. (5). We therefore apply the Bragg-Williams approximation to all of the subsequent analysis.

The overall trend in energy of the hcp phase with increasing Mo concentration obtained from these calculations follows the same behavior that has been reported from other methods. In particular, we have compared our results to those obtained by Kissavos et al. from the coherent potential approximation using the exact muffin-tin orbital solver (EMTO-CPA) for both the bcc and the hcp phases [9, 10], as well as from the special quasirandom structure (SQS) method used by Shin et al., which mimics the cluster correlations of the fully random solid solution in Eq. (4) using a relatively small supercell (16 atoms) [11]. These comparisons are shown in Fig. 1. Deviations between the results of the cluster expansion and the CPA can be attributed partly to differences between DFT methods, particularly with respect to the parameters of the generalized gradient approximation and the type of basis set used. In addition, we note a stronger energy dependence on local relaxations, which are not included in the CPA, in concentration ranges close to the region of mechanical instability. The SQS method, on the other hand, uses the same PAW-PBE framework and allows local relaxations; consequently, the results of the two calculation methods agree almost exactly [11].

Fig. 1 also contains the reported formation enthalpy calculated by Kleykamp from measurements of the free energy at two different temperatures [13]. As has been mentioned in previous theoretical efforts $[9,10]$, the discrepancy between experimental values and the results of $a b$ initio calculations is significant. Whereas those previous studies have attempted to modify the theoretical approach to enforce greater agreement in reported enthalpies, our technique provides new insight into the entropy of formation that allows us to reevaluate these discrepancies, as discussed in the next section. 
In order to model the hcp and bcc phases accurately, fully relaxed structures are necessary over the concentration range of interest. This requirement is complicated by the observed mechanical instability of several structures evaluated at concentrations greater than $x=0.25$ (bcc) or lower than $x=0.6$ (hcp). As an example, three representative structures, whose initial lattices were all of the hcp configuration and whose concentrations are all $x=0.5$, are depicted in Fig. 2, as viewed along the $c$-axis. Whereas the structure in Fig. 2(a) has retained the hexagonal pattern of the unrelaxed lattice, the structures in Figs. 2(b) and 2(c) contain Mo-rich and Ru-rich regions (designated by red and blue spheres, respectively), such that the planes of Mo atoms become unstable and consequently over-relax into the cubic-like substructure.

These structures, and others that by visual inspection do not retain the geometry of the ideal parent lattice upon relaxation, have not been included in the fits of ECI values and are not represented by the points in Fig. 1 because they are not representative of the random solid solution being modeled. While this approach is consistent with the physical system being examined, the exclusion of mechanically unstable structures violates the implicit assumption that the cluster expansion can be applied across all possible configurations. For example, the ECI's in Table I can be applied to the structures in Figs. 2(b) and 2(c) to obtain finite energy values, but because these systems demonstrate observable changes to the ordering of the clusters, the predicted cluster expansion energy is not representative of the true energy of these structures. The result of this approach also implies an extrapolation of the energy derived from Eq. (5) to the unstable pure-composition end members, but these results often do not align with previous CALPHAD assessments [10] and cannot reliably be generalized to other alloy systems. However, by applying the results of the structure inversion only within the concentration range associated with mechanically stable configurations, which is the case for the thermodynamic phase separation of the Mo-Ru system, we can maintain confidence in the results for the disordered mixture providing that unstable configurations represent a very small fraction of the total.

The unit cells used in Fig. 2 are small (6 to 8 atoms) and do not describe the full configurational phase space at $x=0.5$. However, they nonetheless represent a possible metastable state, with a configurational energy close to that of the modeled disordered state. Given the refractory nature of both Mo and $\mathrm{Ru}$ and their correspondingly low mutual diffusivities in solid solutions [34, 35], a nanocomposite of this type, with length scales smaller than the resolution of conventional microscopy techniques, could be stabilized for long periods of time under low-temperature conditions. Consequently, such structures may persist in phase-separated systems with long diffusion length scales $(\sim 10 \mu \mathrm{m})$ even after several weeks of annealing [34-36].

\subsection{Phonon Distributions and Entropy of Formation}

In order to extend the discussion of phase stability beyond purely configurational considerations, a force-constant matrix for each structure was computed by the small-displacement method. The program PHON was then used to calculate the dynamical matrix for each k-point on the $13 \times 13 \times 13$ MonkhorstPack grid and to obtain the associated eigenvalues, which collectively describe the phonon density of states (DOS). Corresponding plots of the DOS are provided in Fig. 3 for hcp Ru and bcc Mo, along with the total and partial DOS for the structure depicted in Fig. 2(a). The distributions for the pure elements agree reasonably well with the reported experimental DOS [37].

In comparison to the two pure-phase plots, the mixture at $x=0.5$ in Fig. 3(b) shows a significant phonon density at lower-energy values $(\sim 5-15 \mathrm{meV})$, which demonstrates an overall softening of the vibrational modes of the system upon mixing. The partial DOS shows that both Mo and Ru exhibit the shift to lower-energy phonon modes, an observation consistent with the bond-proportion model in which Mo$\mathrm{Ru}$ bonding interactions are less stiff and consequently have a smaller spring constant than either Mo-Mo 
or Ru-Ru bonds [21]. The mode observed at $7.6 \mathrm{meV}$, however, is dominated almost entirely by Mo bonding, which we ascribe to the change of the coordination environment between bcc structures ( 8 nearest neighbors) and hcp structures (12 nearest neighbors).

The vibrational contribution to the free energy for a system at temperature $T$ with a known phonon DOS can be written as

$$
F_{v i b}=3 k_{B} T \int_{0}^{\infty} n(v)\left[\frac{h v}{2 k_{B} T}+\ln \left(1-e^{-h v / k_{B} T}\right)\right] d v,
$$

where $k_{B}$ is the Boltzmann constant, $h$ is Planck's constant, and $n(v)$ is the DOS as shown in Fig. 3. In the high-temperature limit ( $T \gg h \bar{v} / k_{B} \approx 300 \mathrm{~K}$ ), Eq. (6) is easily separated into temperature-independent terms that describe the energy and entropy of formation:

$$
\begin{gathered}
\Delta^{f} E_{v i b} \approx \frac{3}{2} h\left(\bar{v}-\bar{v}_{0}\right), \text { and } \\
\Delta^{f} S_{v i b} \approx-3 k_{B} \ln \left(\frac{\overrightarrow{v^{\prime}}}{\vec{v}_{0}^{\prime}}\right),
\end{gathered}
$$

where $\bar{v}$ is the average frequency of the DOS, the subscript 0 represents the concentration-weighted frequencies of the pure phases, and the prime designator indicates a geometric mean, as opposed to the arithmetic mean used for Eq. (7).

In practice, the value of $\Delta^{f} E_{v i b}$, which is controlled by differences in the zero-point energy, is much smaller (on the order of a few meV) than the configurational energy (upwards of $200 \mathrm{meV}$; see Fig. 1). For this reason, vibrational mixing contributions to the energy are sometimes ignored in studies of transition metal alloys. The entropy of formation, however, is controlled by the ratio of mean frequencies, which in turn affects the population of vibrational states. Consequently, small absolute changes in phonon energy may have a significant relative effect on $\Delta^{f} S_{v i b}$.

Fig. 4(a) shows the formation entropy calculated from Eq. (8) for the training set structures in both the bcc and hcp configurations. However, six of the bcc structures were found to give imaginary eigenvalues in calculating the DOS, corresponding to unstable phonon modes that we were not able to resolve through further ionic relaxation. These structures are omitted from the subsequent vibrational analysis.

The overall trend towards higher entropy values as the system approaches equimolar mixing is consistent with the observed phonon softening of Fig. 3. The range of values at $x=0.5$ is approximately $0.3 k_{B}$ to $0.7 k_{B}$, which is the same magnitude as the maximum configurational entropy of a binary alloy, $\Delta^{f} S_{c f g}=k_{B} \ln 2=0.693 k_{B}$. We therefore expect that this increase in entropy will have a significant stabilizing effect on the alloy at high temperatures.

For a comparison of our results to experimental observations, we use reported Debye temperatures of an alloy consisting of increasing Mo concentration in a 5/1/1 mixture of $\mathrm{Ru} / \mathrm{Rh} / \mathrm{Pd}$ [38]. Although this experimental alloy does not exactly match the $\mathrm{Mo} / \mathrm{Ru}$ system described in this work, it nonetheless provides a useful qualitative description of the effect of adding Mo (the only bcc metal in this analysis) into the close-packed environment favored by the other three elements. For the Debye model in the high- 
temperature limit, the vibrational entropy is $\Delta^{f} S_{v i b}=-3 k_{B} \ln \left(\theta / \theta_{0}\right)$, where $\theta$ and $\theta_{0}$ are the mixture and pure-phase Debye temperatures, respectively [39]. The experimental results support a picture of strongly increasing entropy upon mixing.

To complete the comparison to experimental results, we return to the formation energies reported by Kleykamp [13]. In addition to the enthalpy term reproduced in Fig. 1, a total entropy of formation is given in Fig. 4(b) and compared to the sum of vibrational and configurational contributions from our analysis. Here, the vibrational contribution uses the cluster expansion of Eqs. (1) and (5), and the configurational values are given by Eq. (3). As was the case with enthalpy, a significant discrepancy exists between the two results. However, we observe that a negative contribution to the entropy of formation from mixing is highly unusual, and even in the extreme limiting case of complete configurational ordering $\left(\Delta^{f} S_{c f g}=0\right)$, we see no compelling evidence for such a strongly negative vibrational effect. Because both the enthalpy and entropy terms were calculated by a linear fit of two free energy values with a very small relative difference $(\sim 3-5 \%)$ in the original experimental argument, small uncertainties in $\Delta^{f} F$ can easily propagate to large errors in the other reported quantities. Direct comparison of computational results to the experimental free energy of mixing, the subject of the next section, provides a much more suitable test for the validity of the $a b$ initio results.

As a final observation regarding the nature of vibrational mode softening in near-equimolar mixtures, Fig. 4(c) shows the atomic volume of the fully relaxed training structures plotted against the full composition range. These are compared with the Vegard's law approximation, which estimates the volume from a linear mixing relationship of the lattice parameters. For low Ru concentrations, there is slight positive deviation from Vegard's law; however, we estimate that this volume expansion can account for no more than $\sim 10 \%$ of the observed increase in entropy. The remaining entropy increase is likely explained by a combination of the bond-proportion effect and the change of the Mo coordination environment as described above [21].

\subsection{Free Energy and Phase Separation}

The Gibbs free energy can be obtained in the quasiharmonic approximation by minimization with respect to the volume at constant temperature and pressure [21, 39]:

$$
G=\min _{V}\left[E_{c f g}(V)+F_{v i b}(V, T)+P V\right] .
$$

Because the configurational entropy $S_{c f g}$ is independent of $V$, it can be omitted from this expression and reintroduced in the final analysis of the disordered solid solution. For a bulk modulus $B$ obtained from a

quadratic expansion of $E_{c f g}(V)$ and for an observed constant negative value of $\left(\partial F_{v i b} / \partial V\right)_{T}$, the expanded lattice volume used to compute $\mathrm{G}$ is

$$
V=V_{0}\left[1-\frac{\left(\partial F_{v i b} / \partial V\right)_{T}+P}{B}\right],
$$

where $V_{0}$ is the volume of the relaxed lattice from the electronic structure calculation.

The free energies of the training structures were calculated according to Eqs. (9) and (10) for temperatures up to the eutectic temperature of $2200 \mathrm{~K}$, above which melting begins to occur. At each temperature, structure inversions based on Eq. (1) were used to provide ECI's. These values were then 
grouped by cluster order - from the null cluster up to triplets - and added to the values in Table I in order to obtain the necessary parameters for the excess free energy of formation using the form described in Eq. (5). ECI values for both bcc and hcp phases are plotted in Fig. 5 as a function of temperature.

The total free energy of formation is the sum of the quasiharmonic excess term and an ideal term; the latter is determined solely by the configurational entropy in Eq. (3). Combining these terms at $1200 \mathrm{~K}$ gives the curves plotted in Fig. 6 for both bcc and hcp phases. The same calculation is also performed for the simplified harmonic oscillator approximation and for the case in which vibrational terms are neglected altogether in order to demonstrate the significance of both effects in determining the solubility limits in both phases. The quasiharmonic approximation in particular is necessary in order to achieve any appreciable dissolution of Ru within the bcc phase at this temperature.

Comparison of the predicted hcp free energy with the experimental results of Kleykamp, also depicted in Fig. 6 [13], show a greatly improved qualitative agreement relative to the comparisons with the experimentally calculated entropy and enthalpy values, with a minimum in both the theoretical and experimental free energy near $x=0.75$. Although there is still disagreement between theoretical and experimental results with respect to the magnitude of the free energy difference, Cornish and Pratt have reported a similar degree of difficulty in obtaining consistent values for the partial Gibbs energy in the same type of experiment [40].

The last point of comparison explored here between $a b$ initio and experimental results is the binary phase diagram. Because the solubility of $\mathrm{Mo}$ in $\mathrm{Ru}$ (and vice-versa) has been measured directly for many systems, these values have proven highly useful for constraining existing thermodynamic models within the CALPHAD framework [40, 41]. Fig. 7 shows the phase separation calculated by total free energy minimization of the bcc/hcp system, both with and without the quasiharmonic approximation. The $\sigma$ phase, known to appear at high temperatures for Ru concentrations near 38\% [42], is here omitted for simplicity and for comparison to similar metastable phase diagrams reported by Oh et al. and by Cornish and Pratt $[40,41]$.

For temperatures up to $2200 \mathrm{~K}$ (above which the liquid phase begins to appear), the solubility of Mo in the hcp phase from our simulations is mostly within the range of disagreement between the experimental phase diagrams, although the ab initio results tend to deviate from this range at the highest temperatures. Similarly, the bcc phase boundary comparison diverges significantly above $1500 \mathrm{~K}$. At these high temperatures, anharmonic effects beyond the lattice expansion accounted for in the quasiharmonic approach are likely to be much more significant, such that one may encounter a larger number of vibrational energy states than in the harmonic oscillator approximation, leading to a higher entropy and greater phase stability. Additionally, excited electronic states and electron-phonon interactions may begin to play a role in contributing to greater entropy [39].

In the 900-1500 $\mathrm{K}$ temperature range, recent experimental data has shown the solubility, particularly in the bcc phase, to be significantly lower than previously reported values $[35,36]$. As discussed in Section III, this effect may be attributed to the refractory nature of the materials, which necessitates very short length scales between the phases in order to ensure that the alloy material reaches equilibrium [35]. The result of this more recent experimental approach agrees well with the quasiharmonic prediction of the solubility of Ru in the bcc phase. Further investigation of the configurational space at Ru concentrations close to the miscibility gap may also better resolve the exact description of mixing entropy within the cluster expansion, which could account for the remaining differences between computational and experimental results. 


\section{Conclusions}

The magnitude of the contribution of lattice vibrations to free energy estimates in the disordered Mo-Ru system is not inherently obvious. Although significant entropy changes have been observed in order-disorder transitions [21, 43], the formation entropy of binary alloys has received more limited attention. Simulations in the Ni-Cr [44] and Re-W [45] systems using the virtual crystal approximation (VCA) have shown similarly strong increases in $\Delta^{f} S_{v i b}$ commensurate with the configurational entropy. Crucially, these systems, along with the Mo-Ru system described in this work, all exhibit mechanical instabilities in the transition between the bcc structure and close-packed configurations (fcc in the cited cases and hcp in this work). Indeed, the onset of mechanical instability has been directly credited for the large vibrational free energy change, which leads, perhaps counterintuitively, to greater thermodynamic phase stability $[44,46]$.

We have demonstrated a similarly strong phase-stabilizing effect in the alloying of Mo-Ru systems through phonon softening, which contributes to significant vibrational formation entropies in disordered solid solutions. These vibrational contributions are combined with configurational energies through a cluster expansion, which agree well with complementary approximations employed in previous ab initio efforts [9-11]. Total free energies of formation from the combined approach are shown to resolve much of remaining theoretical-experimental gap [13], and predicted solubility limits of the Ru-rich hcp phase and the Mo-rich bcc phase provide a qualitative picture in reasonable agreement with observations [35, 40, 41], particularly at lower temperatures, for which anharmonic effects and electron-phonon interactions are suppressed [39]. The results suggest the need for a thorough treatment of lattice vibrations in any examination of long-range order within the metal particles formed in spent nuclear fuel.

\section{Acknowledgments}

This work was funded by Pacific Northwest National Laboratory utilizing Laboratory Directed Research and Development (LDRD) funds with support from the Nuclear Process Science Initiative (NPSI) and was performed using PNNL Institutional Computing (PIC). Pacific Northwest National Laboratory is operated by Battelle Memorial Institute for the United States Department of Energy under contract DEAC06-76RLO-1830. 
TABLE I. Combined effective cluster interactions $\left(\mathrm{eV}\right.$ atom $\left.{ }^{-1}\right)$ of the disordered configurational energy of formation used in Eq. (5).

\begin{tabular}{ccc}
\hline \hline & bcc & hcp \\
\hline \hline$J^{[0]}$ & 0.1505 & 0.0894 \\
$J^{[1]}$ & 0.0490 & -0.2253 \\
$J^{[2]}$ & -0.0440 & 0.1000 \\
$J^{[3]}$ & 0.0583 & 0.0362 \\
\hline \hline
\end{tabular}




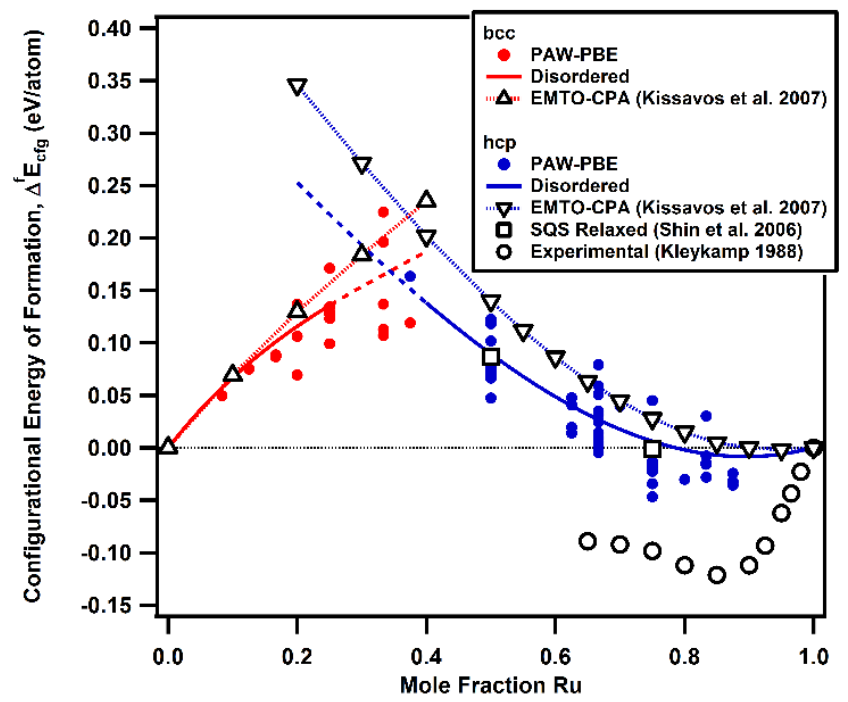

FIG. 1. Calculated configurational contribution to the energy of formation for completely random Mo-Ru alloys in the bcc (red) and hcp (blue) arrangements. Filled circles represent the ordered structures evaluated by DFT as a training set for the cluster expansion. Solid lines depict the predicted energies from the cluster expansion in the disordered case. Comparison is made with the coherent potential approximation (dashed lines and triangles) [9, 10], the special quasirandom structure (open squares) [11], and the reported experimental enthalpy of formation (open circles) [13]. 


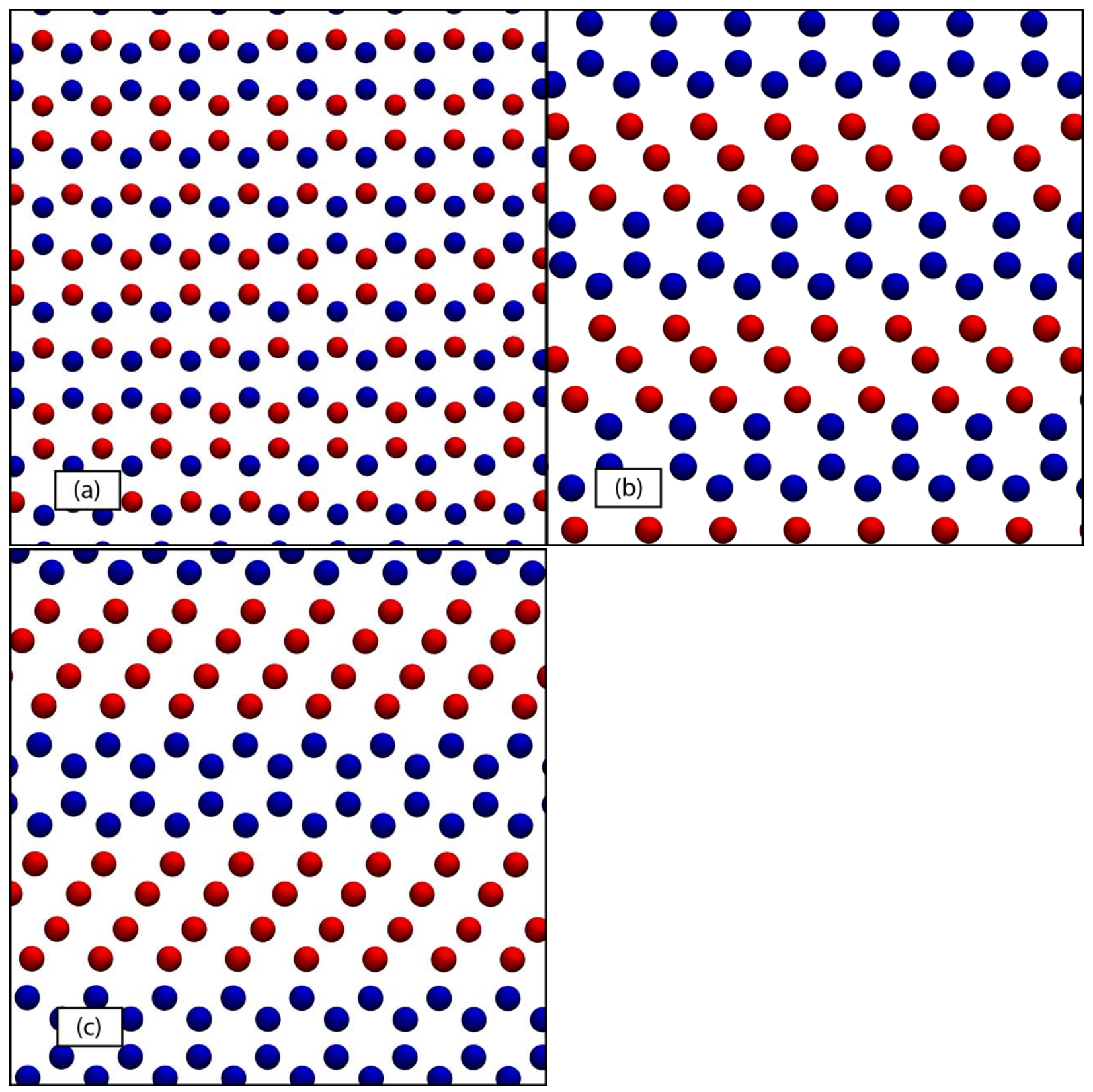

FIG. 2. (a) Sample structure of a 50\%-Ru material, consisting of a 6-atom unit cell, viewed along the $c$ axis. $\mathrm{Ru}$ atoms are in blue; Mo atoms are in red. The hexagonal configuration is preserved upon full relaxation of atom positions. (b) A second structure, also with a 6-atom unit cell at $50 \% \mathrm{Ru}$, showing significant over-relaxation for the Mo-rich region, such that hexagonal long-range order is lost. (c) Overrelaxation effects appearing in a structure defined by an 8 -atom unit cell. 

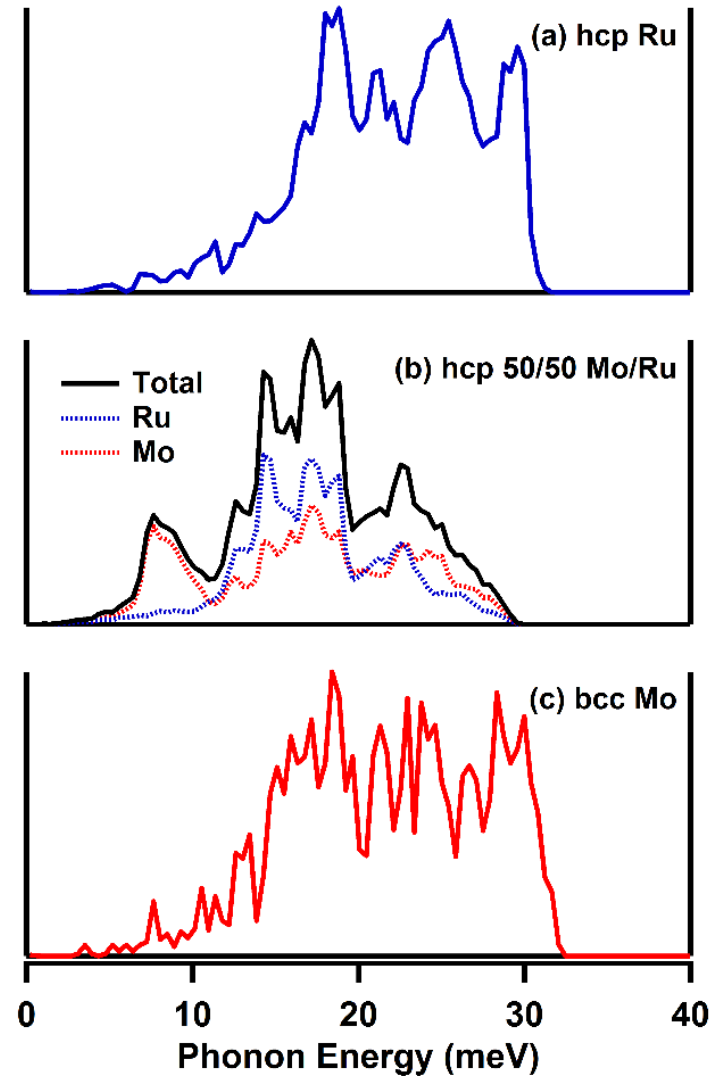

FIG. 3. Phonon density of states for (a) pure hcp Ru, (b) the 50\%-Ru hcp structure shown in Fig. 2(a), and (c) pure bcc Mo. Interaction between $\mathrm{Mo}$ and $\mathrm{Ru}$ atoms leads to a significant phonon softening effect between 5-15 meV, which in turn promotes large vibrational entropy of formation. 

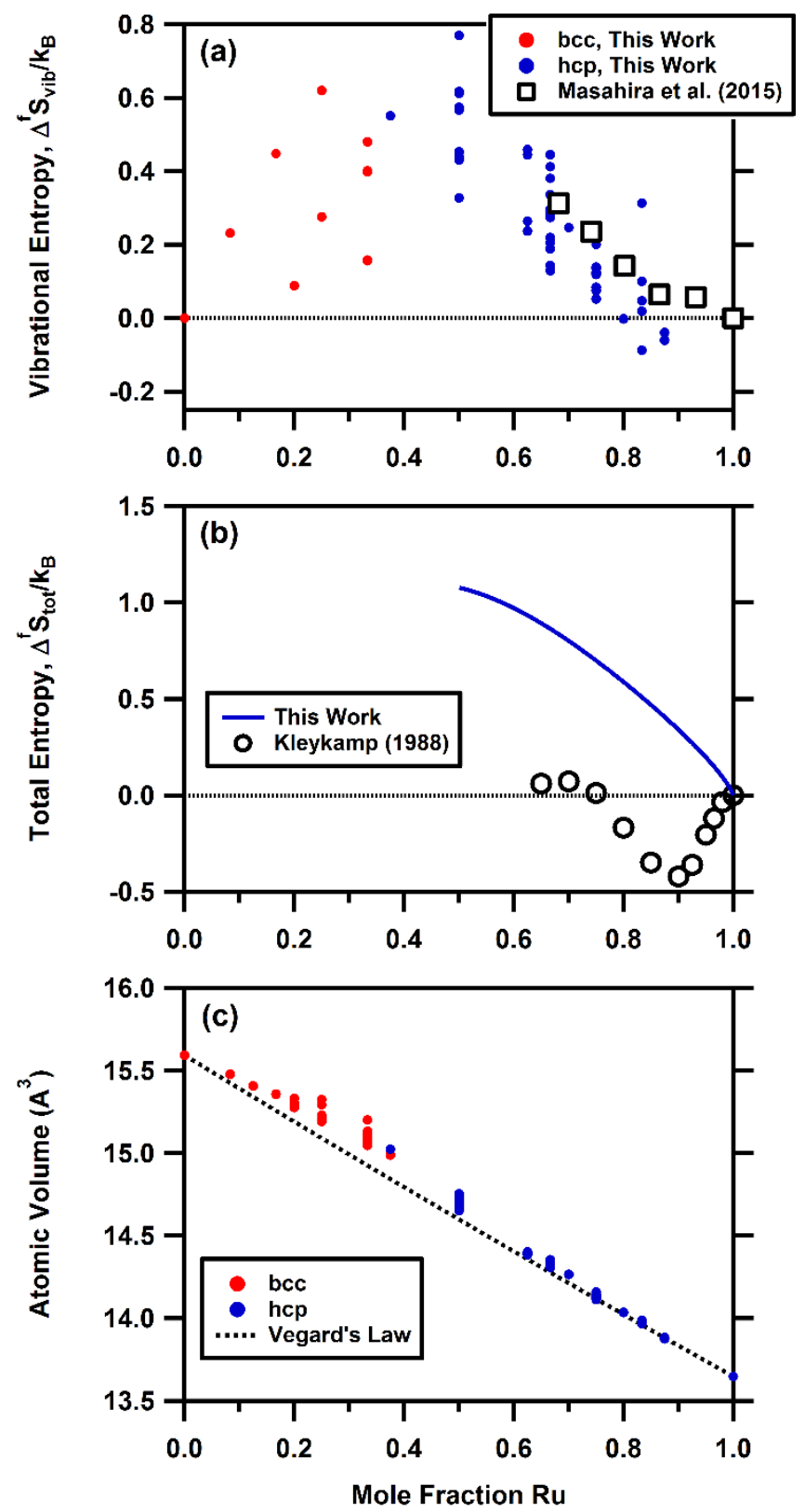

FIG. 4. (a) Vibrational entropy of formation for ordered structures in the cluster expansion (excluding bcc structures with unstable phonon modes). Squares represent entropies derived from reported Debye temperatures [38]. (b) Comparison of the total configurational and vibrational entropy in the hightemperature limit (solid curve) with the reported experimental values of Kleykamp (open circles) [13]. (c) The atomic volume of all ordered structures, showing only modest deviations from Vegard's law. As a result, strong vibrational effects are attributed to the bond-proportion effect. 


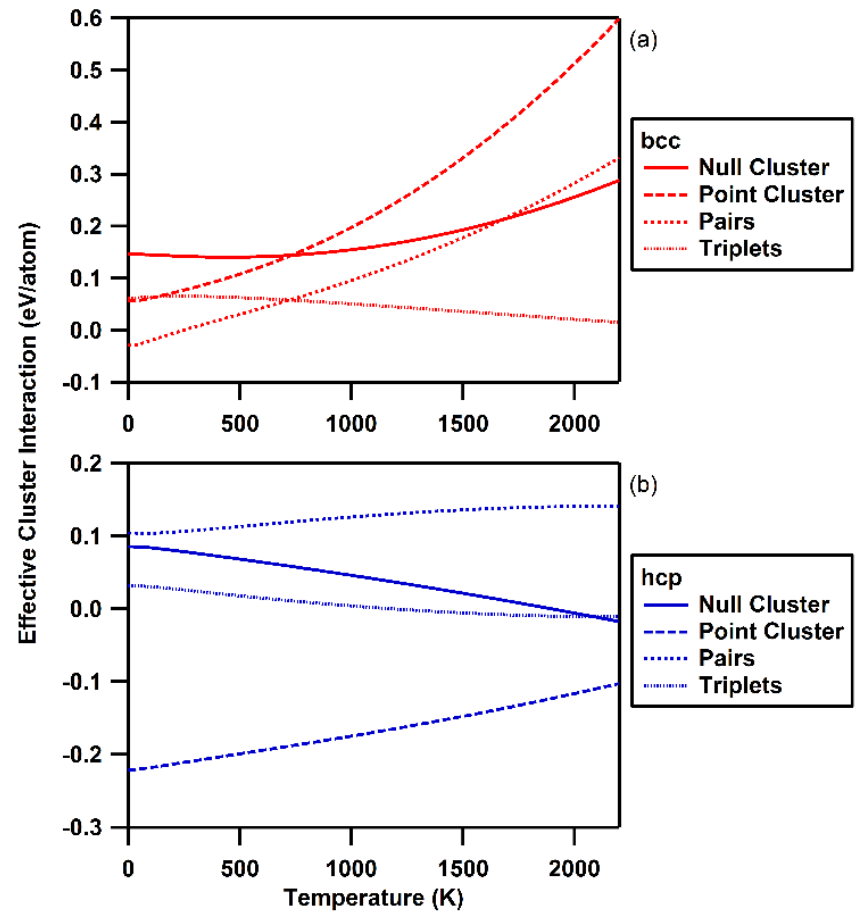

FIG. 5. Combined ECI values as described in Eq. (5) for both (a) bcc and (b) hcp configurations, as a function of temperature. 


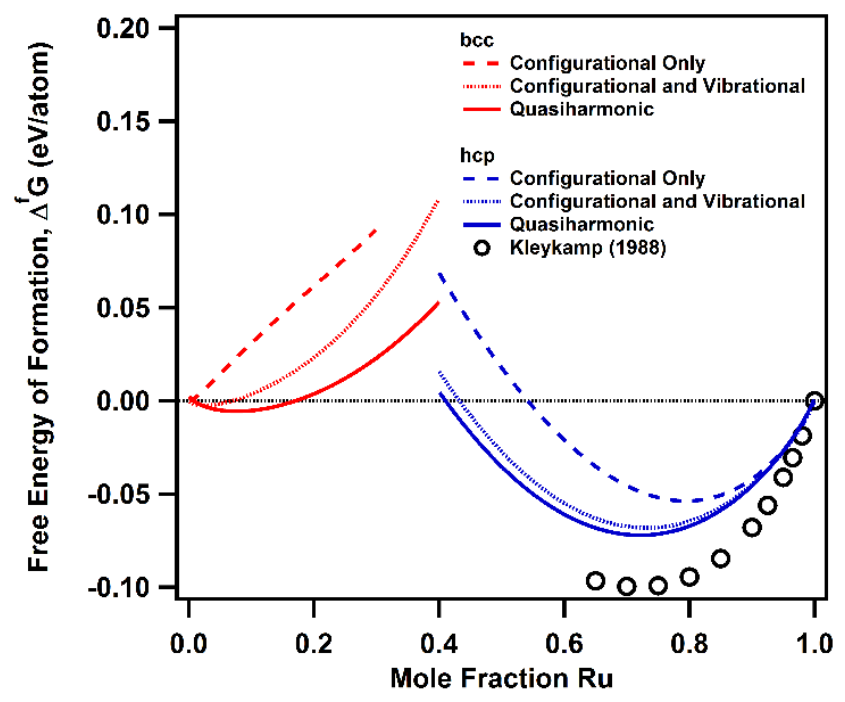

FIG. 6. Comparison of theoretical free energy of mixing in the quasiharmonic approximation (solid curves) with values reported by Kleykamp at $1200 \mathrm{~K}$ (open circles) [13]. There is better qualitative agreement than with the enthalpy (Fig. 1), and both results predict a minimum near $x=0.75$, although some discrepancy remains in estimating the magnitude of the free energy change. Dashed curves show the free energy calculated without the vibrational contribution and demonstrate the need for such effects in accurate descriptions of the phase separation. 


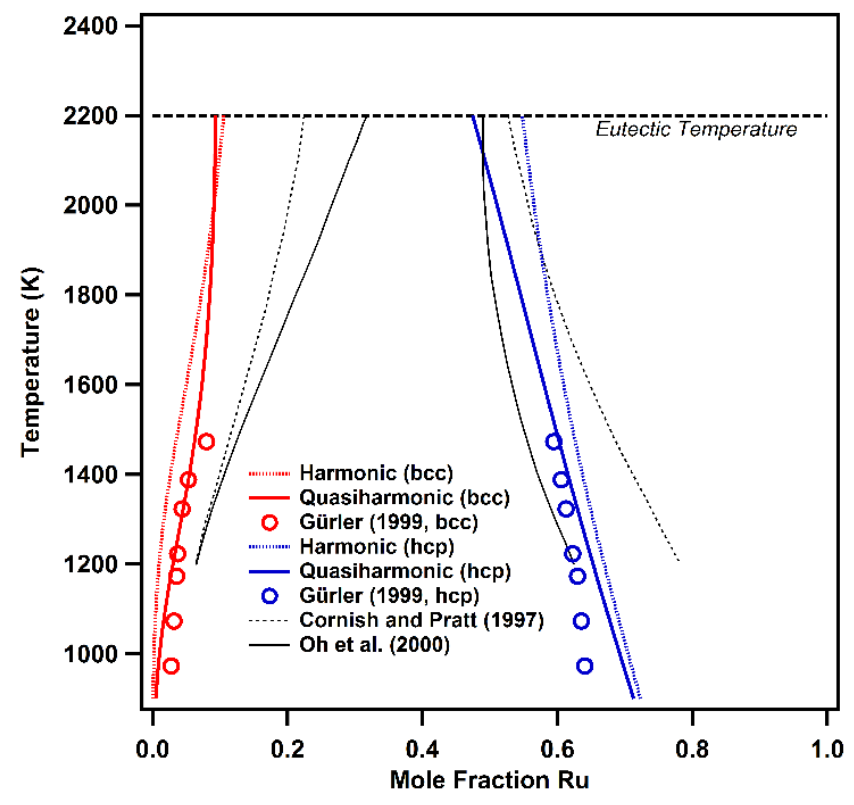

FIG. 7. Metastable phase diagram showing the approximate separation between the bcc and hcp phases (neglecting the high-temperature $\sigma$ phase). Red and blue curves represent the predicted solubility from $a b$ initio calculations, both with and without the quasiharmonic approximation. Solid and dashed black curves are taken from thermodynamic models derived using CALPHAD methodology [40, 41]. Open circles represent the X-ray diffraction measurements made recently by Gürler using smaller diffusion path-lengths than in previous efforts [35].

[1] S. Imoto, Chemical state of fission products in irradiated UO 2, Journal of Nuclear Materials, 140 (1986) 19-27.

[2] H. Kleykamp, The chemical state of the fission products in oxide fuels, Journal of Nuclear Materials, 131 (1985) 221-246.

[3] H. Kleykamp, J. Paschoal, R. Pejsa, F. Thümmler, Composition and structure of fission product precipitates in irradiated oxide fuels: correlation with phase studies in the Mo-Ru-Rh-Pd and BaO-UO 2ZrO 2-MoO 2 Systems, Journal of Nuclear Materials, 130 (1985) 426-433.

[4] H. Kleykamp, Post-irradiation examinations and composition of the residues from nitric acid dissolution experiments of high-burnup LWR fuel, Journal of Nuclear Materials, 171 (1990) 181-188.

[5] H. Kleykamp, R. Pejsa, X-ray diffraction studies on irradiated nuclear fuels, Journal of nuclear materials, 124 (1984) 56-63.

[6] J. Bramman, R. Sharpe, D. Thom, G. Yates, Metallic fission-product inclusions in irradiated oxide fuels, Journal of Nuclear Materials, 25 (1968) 201-215.

[7] F. Ducastelle, Order and phase stability in alloys, (1991).

[8] A. Van der Ven, M. Aydinol, G. Ceder, G. Kresse, J. Hafner, First-principles investigation of phase stability in Li x CoO 2, Physical Review B, 58 (1998) 2975.

[9] A.E. Kissavos, S. Shallcross, L. Kaufman, O. Grånäs, A.V. Ruban, I.A. Abrikosov, Thermodynamics of ordered and disordered phases in the binary Mo-Ru system, Physical Review B, 75 (2007) 184203.

[10] A.E. Kissavos, S. Shallcross, V. Meded, L. Kaufman, I.A. Abrikosov, A critical test of ab initio and CALPHAD methods: The structural energy difference between bcc and hcp molybdenum, Calphad, 29 (2005) 17-23. 
[11] D. Shin, R. Arróyave, Z.-K. Liu, A. Van de Walle, Thermodynamic properties of binary hcp solution phases from special quasirandom structures, Physical Review B, 74 (2006) 024204.

[12] S. Middleburgh, D. King, G. Lumpkin, Atomic scale modelling of hexagonal structured metallic fission product alloys, Royal Society open science, 2 (2015) 140292.

[13] H. Kleykamp, Thermodynamics of the Mo • Ru system, Journal of the Less Common Metals, 144 (1988) 79-86.

[14] P.E. Turchi, I.A. Abrikosov, B. Burton, S.G. Fries, G. Grimvall, L. Kaufman, P. Korzhavyi, V.R. Manga, M. Ohno, A. Pisch, Interface between quantum-mechanical-based approaches, experiments, and CALPHAD methodology, Calphad, 31 (2007) 4-27.

[15] J. Connolly, A. Williams, Density-functional theory applied to phase transformations in transitionmetal alloys, Physical Review B, 27 (1983) 5169.

[16] J. Sanchez, Cluster expansions and the configurational energy of alloys, Physical review B, 48 (1993) 14013.

[17] J. Sanchez, Cluster expansion and the configurational theory of alloys, Physical Review B, 81 (2010) 224202.

[18] J.M. Sanchez, F. Ducastelle, D. Gratias, Generalized cluster description of multicomponent systems, Physica A: Statistical Mechanics and its Applications, 128 (1984) 334-350.

[19] J. Sanchez, J. Stark, V. Moruzzi, First-principles calculation of the Ag-Cu phase diagram, Physical Review B, 44 (1991) 5411.

[20] G. Ceder, A derivation of the Ising model for the computation of phase diagrams, Computational Materials Science, 1 (1993) 144-150.

[21] A. Van De Walle, G. Ceder, The effect of lattice vibrations on substitutional alloy thermodynamics, Reviews of Modern Physics, 74 (2002) 11.

[22] A. Van De Walle, G. Ceder, First-principles computation of the vibrational entropy of ordered and disordered Pd 3 V, Physical Review B, 61 (2000) 5972.

[23] A. Van de Walle, G. Ceder, U. Waghmare, First-principles computation of the vibrational entropy of ordered and disordered Ni 3 Al, Physical review letters, 80 (1998) 4911.

[24] A. van de Walle, Multicomponent multisublattice alloys, nonconfigurational entropy and other additions to the Alloy Theoretic Automated Toolkit, Calphad, 33 (2009) 266-278.

[25] A. Van de Walle, M. Asta, G. Ceder, The alloy theoretic automated toolkit: A user guide, Calphad, 26 (2002) 539-553.

[26] A. van de Walle, G. Ceder, Automating first-principles phase diagram calculations, Journal of Phase Equilibria, 23 (2002) 348-359.

[27] A. Van de Walle, M. Asta, Self-driven lattice-model Monte Carlo simulations of alloy thermodynamic properties and phase diagrams, Modelling and Simulation in Materials Science and Engineering, 10 (2002) 521.

[28] G. Kresse, J. Furthmüller, Efficient iterative schemes for \textit\{ab initio\} total-energy calculations using a plane-wave basis set, Physical Review B, 54 (1996) 11169-11186.

[29] G. Kresse, J. Furthmüller, Efficiency of ab-initio total energy calculations for metals and semiconductors using a plane-wave basis set, Computational Materials Science, 6 (1996) 15-50.

[30] P.E. Blöchl, Projector augmented-wave method, Physical Review B, 50 (1994) 17953-17979.

[31] J.P. Perdew, K. Burke, M. Ernzerhof, Generalized Gradient Approximation Made Simple, Physical Review Letters, 77 (1996) 3865-3868.

[32] H.J. Monkhorst, J.D. Pack, Special points for Brillouin-zone integrations, Physical Review B, 13 (1976) 5188-5192.

[33] D. Alfè, PHON: A program to calculate phonons using the small displacement method, Computer Physics Communications, 180 (2009) 2622-2633. 
[34] H. Kleykamp, The constitution of the Mo $\cdot$ Ru system, Journal of the Less Common Metals, 136 (1988) 271-275.

[35] R. Gürler, Constitutional studies of molybdenum-ruthenium alloys using ultra-rapidly solidified samples, Journal of alloys and compounds, 285 (1999) 133-136.

[36] E. Anderson, W. Hume-Rothery, The equilibrium diagram of the system molybdenum-ruthenium, Journal of the Less Common Metals, 2 (1960) 443-450.

[37] K. Hellwege, J. Olsen, Phonon states of elements Electron states and Fermi Surfaces of Alloys vol 13a, in, Berlin: Springer) p, 1981.

[38] Y. Masahira, Y. Ohishi, K. Kurosaki, H. Muta, S. Yamanaka, S. Komamine, T. Fukui, E. Ochi, Effect of Mo content on thermal and mechanical properties of Mo-Ru-Rh-Pd alloys, Journal of Nuclear Materials, 456 (2015) 369-372.

[39] B. Fultz, Vibrational thermodynamics of materials, Progress in Materials Science, 55 (2010) 247-352. [40] L. Cornish, J. Pratt, Constitutional studies of the molybdenum-ruthenium-palladium ternary system, Journal of alloys and compounds, 247 (1997) 66-71.

[41] C.-S. Oh, H. Murakami, H. Harada, Thermodynamic evaluation of the Mo-Ru system, Journal of alloys and compounds, 313 (2000) 115-120.

[42] O. Grånäs, P.A. Korzhavyi, A. Kissavos, I.A. Abrikosov, Theoretical study of the Mo-Ru sigma phase, Calphad, 32 (2008) 171-176.

[43] B. Fultz, L. Anthony, L. Nagel, R. Nicklow, S. Spooner, Phonon densities of states and vibrational entropies of ordered and disordered Ni 3 Al, Physical Review B, 52 (1995) 3315.

[44] P. Craievich, J. Sanchez, Vibrational free energy in the Ni-Cr system, Computational materials science, 8 (1997) 92-99.

[45] K. Persson, M. Ekman, G. Grimvall, Dynamical and thermodynamical instabilities in the disordered Re x W 1- x system, Physical Review B, 60 (1999) 9999.

[46] P. Craievich, J. Sanchez, R. Watson, M. Weinert, Structural instabilities of excited phases, Physical Review B, 55 (1997) 787. 